\title{
Pitfalls in microcystin extraction and recovery from human blood serum
}

\author{
Alexandra H. Heussner ${ }^{\mathrm{a}, 1}$, Stefan Altaner ${ }^{\mathrm{a}, 1}$, Lisa Kamp ${ }^{\mathrm{b}}$, Fernando Rubio ${ }^{\mathrm{b}}$, Daniel R. Dietrich ${ }^{\mathrm{a}, *}$ \\ ${ }^{a}$ Human and Environmental Toxicology, University of Konstanz, Universitätsstraße 10, 78457 Konstanz, Germany \\ ${ }^{\mathrm{b}}$ Abraxis LLC, 54 Steamwhistle Drive, Warminster, PA 18974, USA
}

Keywords:

ELISA

Microcystin

Human serum

Recovery

Adsorption to lab ware

Hydrophobicity

\begin{abstract}
A B S T R A C T
Background: Microcystins (MCs) contaminate water bodies due to cyanobacterial blooms all over the world, leading to frequent exposure of humans to MCs through consumption of meat, fish, seafood, blue green algal products and water, accidental ingestion of contaminated water and scum during recre ational activities and inhalation of cyanobacterial aerosols. For monitoring of human exposure, sensitive screening methods are needed. However, during the analytical process of various matrices, such as human serum, some problems appear to regularly occur during sample preparation and storage, leading to MC loss and thus to underestimation of the true MC concentration. The aim of the current study was therefore to assess the pitfalls of the MC extraction method from human serum with more detail. Methods: Six MC congeners (MC LR, YR, RR, LA, LW, LF) and defined equimolar MC mixtures thereof were spiked into human serum, and quantified using the commercially available Adda ELISA subsequent to standard extraction (methanol extraction with subsequent SPE). To detect the potential influence of sample storage and preparation/storage materials, different types of material such as glass, standard polypropylene and surface treated polypropylene were compared.

Results: Loss of MC during preparation and storage is largely dependent on (1) the handling of the stored material, (2) the 'surface' of the storage material and (3) the hydrophobicity of the MCs.

Conclusions: The pitfalls described for MC analysis with the ELISA are primarily associated with sample preparation and clean up and thus also apply to other analytical techniques for MC detection beyond the ELISA used. It can be concluded that ELISA based methods are suitable tools for the detection of MCs in human sera and other samples.
\end{abstract}

\section{Background}

Microcystins (MCs) are toxic cyclic peptides produced by cer tain cyanobacterial genera such as Anabaena, Microcystis, Nostoc and Planktothrix that contaminate water bodies due to cyanobac terial blooms all over the world [1]. This leads to the frequent exposure of humans to MCs through consumption of meat, fish, seafood, blue green algal products and water, accidental ingestion of contaminated water and cyanobacterial scum during recrea tional activities and inhalation of cyanobacterial aerosols [2 5$]$.

Abbreviations: $\mathrm{MeOH}$, methanol; SPE, solid phase extraction; MC, microcystin; LOD, limit of detection; RT, room temperature; HPLC, high performance liquid chromatography.

* Corresponding author. Tel.: +49753188 3518.

E-mail addresses: alexandra.heussner@uni-konstanz.de (A.H. Heussner), stefan. altaner@uni-konstanz.de (S. Altaner), lkamp@abraxiskits.com (L. Kamp), frubio@ abraxiskits.com (F. Rubio), daniel.dietrich@uni-konstanz.de (D.R. Dietrich).

${ }^{1}$ These authors contributed equally to the publication.
In order to monitor human exposure, sensitive screening methods are needed. In the past, ELISA has been demonstrated to be a robust routine tool for MC quantification in environmental samples and increasingly ELISAs are also used to detect MCs in tissue and blood samples from various species including humans [6 11]. However, some problems regularly occur during sample preparation, leading to $\mathrm{MC}$ loss and therefore to underestimation of the true MC con centration $[2,1116]$.

The aim of the current study was to assess the pitfalls of the MC extraction from complex media with more detail. For this, MCs (MC LR, YR, RR, LA, LW, LF and defined equimolar MC mixtures thereof) were spiked into human blood serum as a surro gate for very complex sample media and quantified using the com mercially available Adda ELISA. To detect the potential influence of sample storage and preparation materials, the effect of different types of material (i.e. glass, standard polypropylene and surface treated polypropylene) on MC recovery were compared. 


\section{Materials and methods}

\subsection{Materials}

Unless otherwise stated, materials were purchased as follows: Abraxis, Warminster, PA, USA (ELISA kit), Waters GmbH, Eschborn, Germany (Oasis ${ }^{\circledR}$ HLB 6 cc (200 mg) extraction cartridges), Enzo Life Sciences GmbH, Lörrach, Germany (MC LR, MC YR, MC RR, MC LA, MC LW and MC LF), Carl Roth, Karlsruhe, Germany (Roti labo ${ }^{\circledR}$ screw neck ND8 vials (clear and brown glass $1.5 \mathrm{~mL}$ ) with Butyl/PTFE seal in screw cap, cat\# KE26.1, KE30.1, TY73.1; yellow pipette tips, cat\# 2395.1; cristal pipette tips, cat\# 2393.1), Eppen dorf, Wesseling Berzdorf, Germany (Protein LoBind tube $2.0 \mathrm{~mL}$, cat\# $0030108.132 ; 300 \mu \mathrm{L}$ pipette tips (for multichannel pipet ting), cat\# 022492047; blue pipette tips, cat\# 0030000919), Sar stedt, Nümbrecht, Germany (micro tube $1.5 \mathrm{~mL}$ low binding, cat\# 72.706.600; standard PP micro tube $1.5 \mathrm{~mL}$, cat\# 72.690.001; $5 \mathrm{~mL}$ pipette tips, cat\# 70.1183.002), Biozym Scientific, Hessisch Oldendorf, Germany (micro tube $2.0 \mathrm{~mL}$, clear, low binding, cat\# 71028), Corning, Kaiserslautern, Germany (MAXYMum Recovery microtube $1.7 \mathrm{~mL}$, cat\# MCT $175 \mathrm{~L}$ C), and Sigma Aldrich GmbH, Seelze, Germany (all other chemicals and materials).

\subsection{Preparation and storage of samples}

MC LR and MC RR were dissolved in $100 \% \mathrm{MeOH}$ in glass vials to a nominal concentration of $100 \mu \mathrm{M}$ using vigorous mixing, shak ing for $30 \mathrm{~min}$ at $4{ }^{\circ} \mathrm{C}$ and sonication $(5 \mathrm{~min}$ ). The real concentra tion was determined via detection of absorbance at $\lambda_{238 \mathrm{~nm}}$ and using the molar absorption coefficient of $39,800 \mathrm{~mol} \mathrm{~L}^{-1} \mathrm{~cm}^{-1}$

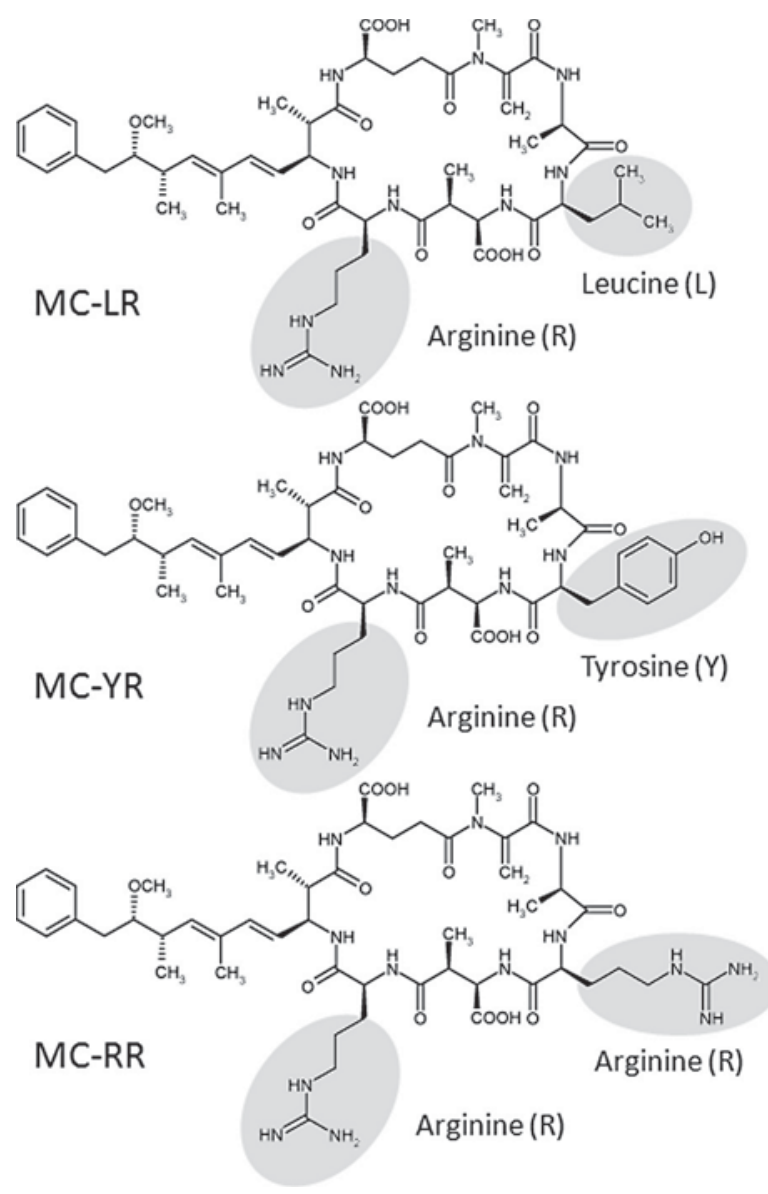

[17] after calibration of the spectrophotometer with potassium dichromate in $1 \mathrm{mM}$ perchloric acid [18]. Due to lack of validated coefficients for each of the MC congeners used in this study, all MC congeners were prepared as if they had the same absorption coefficient as MC LR. Although some investigators used specific extinction coefficients (Wayne Carmichael, personal communica tion), using the same coefficient for the major MC congeners repre sents a widely accepted approach because the differences seem to be quite small (personal communication with Jussi Meriluoto and Linda Lawton) and thus will not impact the overall findings (for a more detailed discussion of this issue see $[11,19])$. The structural differences among the six MC congeners employed in this study are depicted in Fig. 1.

Dilution series of MCs were performed in glass vials. Human serum was spiked with the different MC congeners $(1100 \mu \mathrm{g} / \mathrm{L})$ or a defined equimolar mixture of them (total of 1,10 or $100 \mu \mathrm{g} /$ L) in glass vials. Samples thus generated were then distributed to glass vials, standard polypropylene (PP) tubes or 'low binding' PP tubes for the detection of handling and storage effects of the materials (Fig. 2). Samples were stored short term at $-20^{\circ} \mathrm{C}$ until further use.

\subsection{Extraction and purification of toxins from human serum}

Spiked and control human serum samples were submitted to a methanol/hexane extraction as described by Hilborn and co work ers with some modifications [10], see Fig. 2. Subsequent to the organic solvent extraction and clean up, solid phase extraction (SPE) was performed using Oasis ${ }^{\circledR}$ HLB 6 cc (200 mg) cartridges, according to the manufacturer's instructions (Waters $\mathrm{GmbH}$,
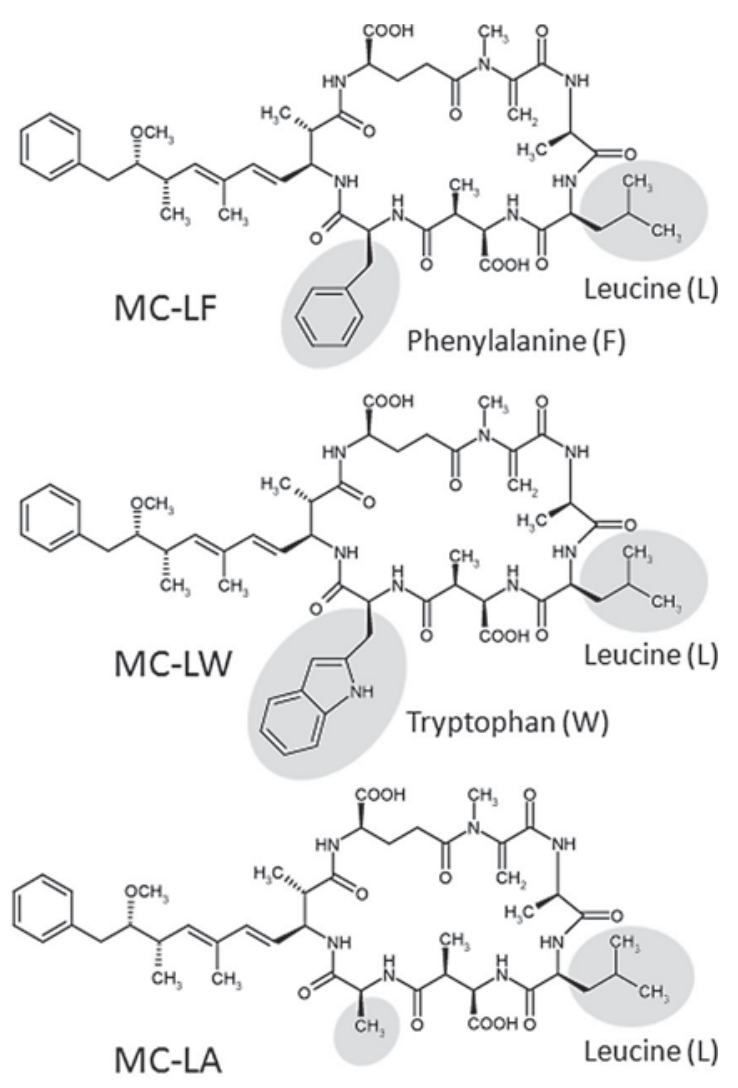

Alanine (A)

Fig. 1. Structures of MC congeners used in this study. The variable amino acids (labeled in gray), largely determining the relative hydrophobicity of the whole molecule, are also reflected in the name and abbreviation of the respective MC-congener. 


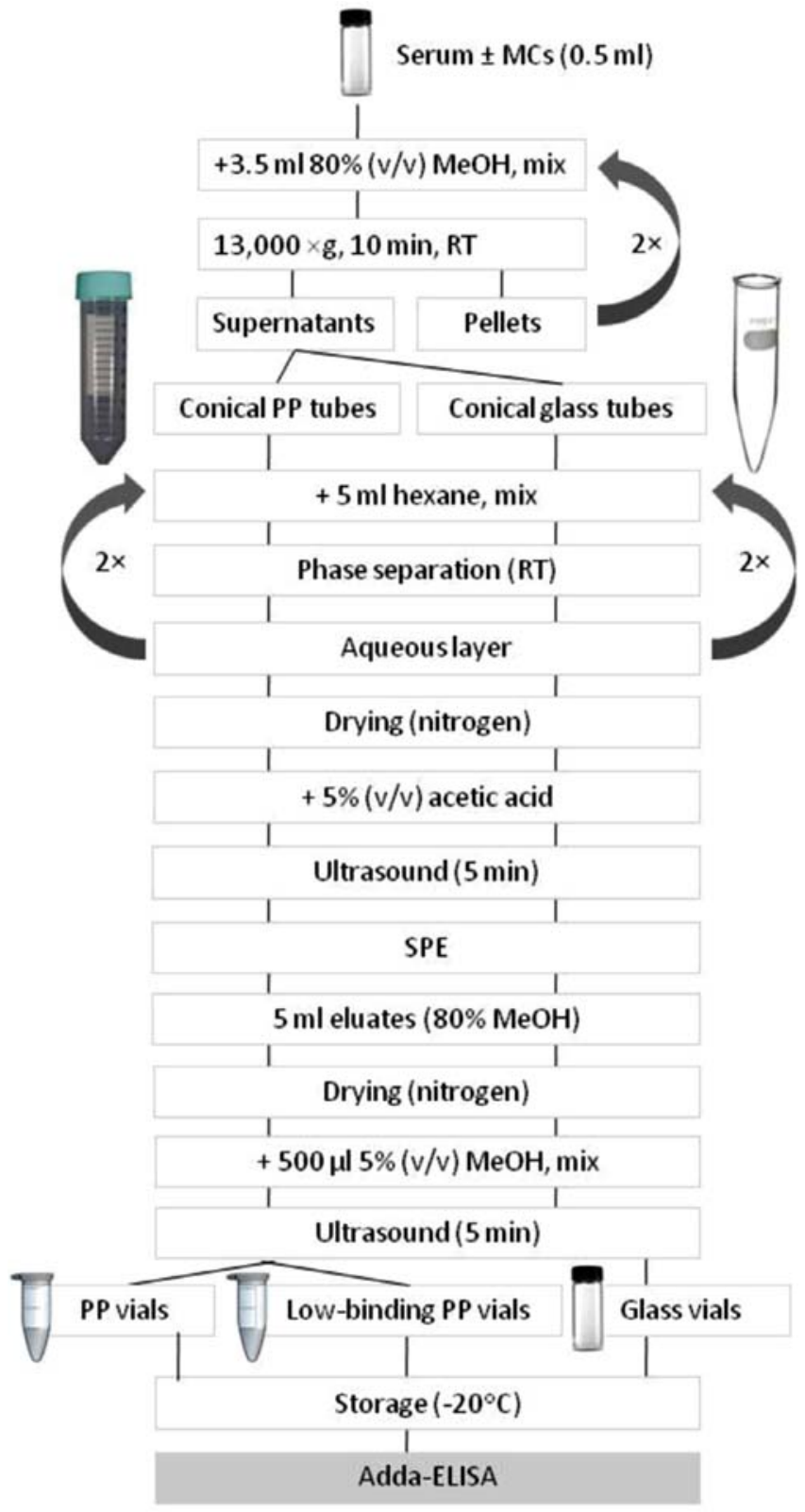

Fig. 2. Overview of the sample preparation procedure.

Eschborn, Germany), in order to further purify the toxins in the extracts. Briefly, SPE columns were preconditioned with $100 \%$ $\mathrm{MeOH}(2 \mathrm{~mL})$ and equilibrated with deionized water $(2 \mathrm{~mL})$. Serum extracts were applied to the SPE columns and the columns were washed with $30 \%(\mathrm{v} / \mathrm{v}) \mathrm{MeOH}$ in $0.1 \mathrm{M}$ acetic acid $(3 \times 2 \mathrm{~mL}) .80 \%$ $\mathrm{MeOH}(5 \mathrm{~mL})$ was employed for MC elution from the column into either polypropylene (PP), low binding PP or glass tubes. Pooled eluates from the same sample were evaporated to dryness under a stream of nitrogen. Dried samples were resuspended in $500 \mu \mathrm{L}$ $5 \%(\mathrm{v} / \mathrm{v})$ aqueous $\mathrm{MeOH}$, vigorously mixed and sonicated for $5 \mathrm{~min}$. These samples were then stored at $-20^{\circ} \mathrm{C}$ for 0 (no storage), 1 and 2 months prior to analysis as described in detail below.

\subsection{Adda ELISA}

Toxin extracts were analyzed using a commercially available Adda ELISA kit (Microcystins/Nodularins (ADDA) ELISA, Abraxis LLC, Warminster, PA, USA; cat\# 520011) according to manufac turer's instructions. This indirect competitive ELISA recognizes specifically the Adda moiety present in all known MC congeners via a polyclonal antibody, thus detecting MCs independent of the MC congener [20]. Each individual sample was analyzed in dupli cate on each plate (technical replicates), whereby three indepen dent samples (true replicates) were generated for every sample for all analyses. Prior to Adda ELISA analyses, extracts were diluted to nominally $1 \mu \mathrm{g} \mathrm{MC} / \mathrm{L}$ in order to fit the presumed MC content to the linear range of the Adda ELISA $(0.155 .0 \mu \mathrm{g} / \mathrm{L})$. MC LR equiva lent concentrations $\left(M C \mathrm{LR}_{\mathrm{eq}}\right.$ ) were calculated from the MC LR stan dard calibration curve. Extracts that were below the range of the standard curve of each individual ELISA run were considered to be below the limit of detection ( $<$ LOD of $0.15 \mu \mathrm{g} / \mathrm{L}$ MC LR) of the Adda ELISA used. Based on the detectability of the various MC cong eners in the ELISA and the reported coefficients of variation (CVs) for standards (CV < 10\%) and samples (CV < 15\%), expected theoretical range of MC recovery was set ranging between $80 \%$ and $120 \%$. The latter is supported by our preliminary data, CVs and observations by the manufacturer and earlier reports $[11,20,21]$.

\subsection{Calculations and statistical data analysis}

Adda ELISA data were processed according to manufacturer's instructions. Briefly, absorbance values were calculated as percent age of the control for each plate and compared with the calibration data achieved via linear regression analysis. Means \pm SDs were cal culated from at least three independent samples (true replicates) conducted in duplicate (technical replicates). Recovery data were compared to a theoretical $100 \pm 20 \%$ recovery by One way ANOVA with Dunnett's Multiple Comparison Test.

Data are presented as means \pm SEM of three individual experi ments $(n=3)$, if not stated otherwise. If individual replicates of one sample were out of range of the standard curve during an Adda ELISA analysis, these samples were omitted from further sta tistical analyses and the final true replicate number reported as $n=2$ or $n=1$. Significant differences are indicated as follows: $p<0.05(*), p<0.01(* *)$ and $p<0.001(* * *)$. All statistical analyses were performed using GraphPad Prism 5.03 software.

\section{Results}

3.1. Effect of storage time and materials on the recovery of MC LR from human blood serum

The recovery of MC LR from human serum was investigated using two different storage vessels to determine potential toxin loss due to adsorption to the vessels. For this, human serum was spiked with MC LR at different concentrations (1 $100 \mu \mathrm{g} / \mathrm{L})$, extracted in either glass vials or standard PP tubes and then stored at $-20^{\circ} \mathrm{C}(0,1$ or 2 months $)$ in the respective vessels. MC LR recov ery was analyzed using the Adda ELISA. Contrary to expectations, no difference was observed between MC LR concentrations, stor age times and type of vessel material used (Fig. 3). A significant dif ference from the theoretical range of $80120 \%$ recovery was detected in only one sample $(1 \mu \mathrm{g} / \mathrm{L}, 2$ months storage in glass vials, Fig. 3A), however this difference was considered an experi mental artifact when compared with the complete data set for the same vessel material. The same recovery results were obtained with MC LR concentrations up to $1000 \mu \mathrm{g} / \mathrm{L}$ (data not shown).

3.2. Effect of storage time and materials on the recovery of six MC congeners from human blood serum

The $\mathrm{MC}$ recovery from spiked human serum using six different MC congeners and three concentrations $(1,10,100 \mu \mathrm{g} / \mathrm{L})$ as well as an equimolar mix of the six MC congeners with final concentrations 

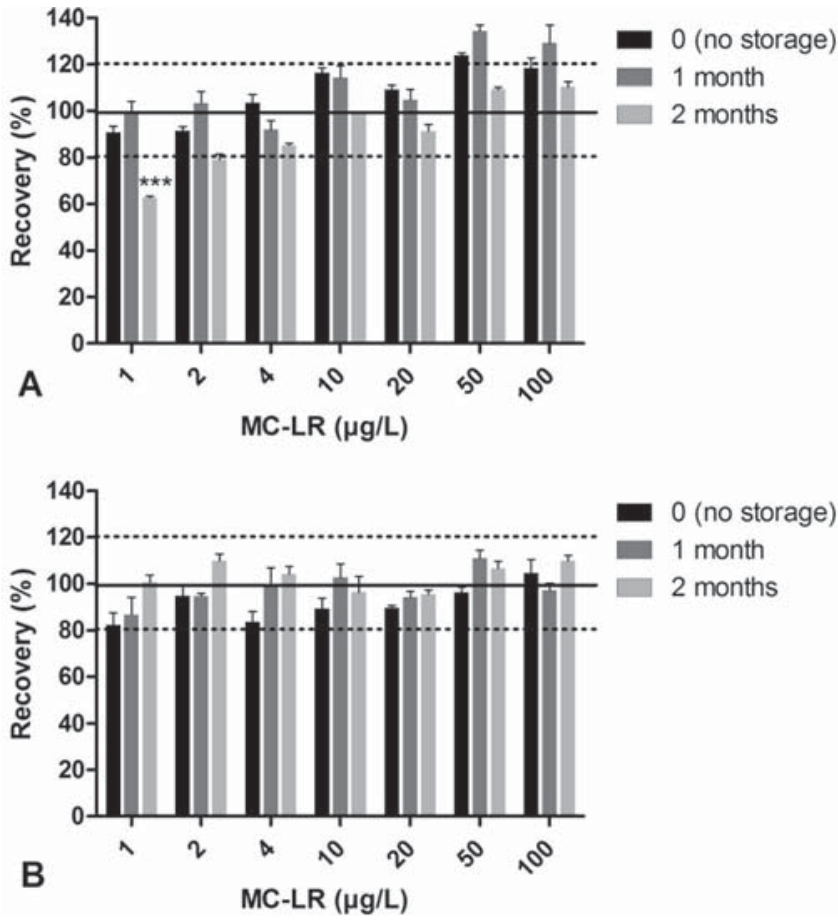

Fig. 3. Recovery of MC-LR from spiked serum using the Adda-ELISA. Serum samples were spiked with varying concentrations of MC-LR, subjected to organic extraction in either glass (A) or in standard polypropylene vials (B), followed by an SPE-cleanup, and storage of the extracts in $5 \% \mathrm{MeOH}$ for varying durations at $-20{ }^{\circ} \mathrm{C}$ in either glass (A) or in polypropylene vials (B) and then submitted to Adda-ELISA analysis. Results are compared to a theoretical recovery range $(100 \pm 20 \%)$. Columns represent means from three independent samples $(n=3) \pm$ SEM; dotted lines represent the theoretical recovery range (80-120\%). One-way ANOVA with Dunnett's multiple comparison test with $p<0.001(* * *)$.

of 1,10 or $100 \mu \mathrm{g} / \mathrm{L}$ was determined for two different storage vials (glass and standard PP tubes) and storage times of 0 (no storage), 1 and 2 months (Fig. 4A C).

MC LR, YR and RR were recovered completely from the glass vials, irrespective of the originally spiked MC concentration and the storage time. In contrast, no acceptable recovery was achieved for MC LA, LW and LF. However, while nearly acceptable recover ies were achieved when samples were immediately analyzed, recoveries declined with length of storage time and also inverse to the nominal concentration tested (Fig. 4A C). Similarly, recover ies obtained with the MC mixtures suggested acceptable results when immediately tested and lower recoveries upon storage, albeit a trend with storage time was not observable.

The identical approach applied to standard PP vials resulted in a similar picture as observed for the glass vials (Fig. 5A C), although the recovery of MC RR at the $1 \mu \mathrm{g} / \mathrm{L}$ concentrations was below the acceptable recovery irrespective of the storage duration. In con trast, storage of MC LR and YR did not appear to affect recoveries (Fig. 5A C). As expected, the more hydrophobic MC congeners LA, LW and LF were not recoverable at the 1 and $10 \mu \mathrm{g} / \mathrm{L}$ concentra tions employed irrespective of the duration of storage, while at least for MC LA approximately 50\% was recovered at the $100 \mu \mathrm{g}$ / $\mathrm{L}$ level (Fig. 5A C). The MC mixtures mirrored the findings for the single toxins, whereby minimal recovery was achieved only for 10 and $100 \mu \mathrm{g} / \mathrm{L}$ mixtures.

\section{3. $M C$ recovery from 'low binding' $P P$ vials}

For practical reasons, plastic labware is more convenient than glassware. Therefore, various specialized plastics were tested for
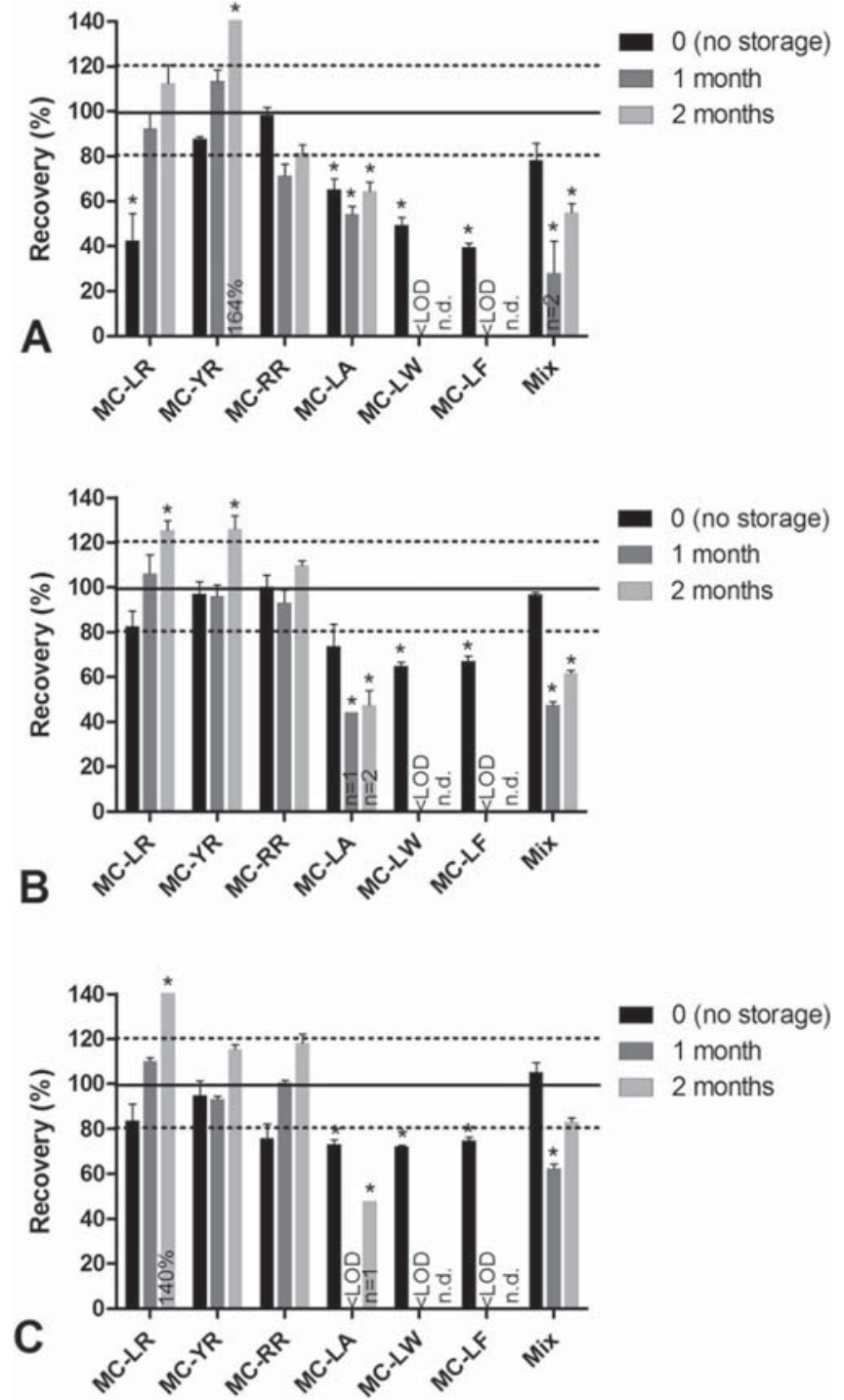

Fig. 4. Recovery of six MC congeners from spiked human serum (glass vials) using the Adda-ELISA. Human serum was spiked with $1 \mu \mathrm{g} / \mathrm{L}(\mathrm{A}), 10 \mu \mathrm{g} / \mathrm{L}$ (B) and $100 \mu \mathrm{g} / \mathrm{L}$ (C) of each MC congener or an equimolar mixture of the six MC congeners to a final MC mix concentration of $1 \mu \mathrm{g} / \mathrm{L}(\mathrm{A}), 10 \mu \mathrm{g} / \mathrm{L}$ (B) and $100 \mu \mathrm{g} / \mathrm{L}$ (C). Glass vials were used for storage $\left(-20^{\circ} \mathrm{C}\right)$ and processing. Columns represent means from three independent samples ( $n=3$ unless stated otherwise) + SEM; dotted lines represent the recovery range (80-120\%). One-way ANOVA with Dunnett's multiple comparison test with $p<0.05(*)$. LOD: Limit of detection $=0.15 \mu \mathrm{g} / \mathrm{L}$; n.d., not determined.

their suitability for handling and storage in MC analysis. Several plastic labware suppliers provide so called 'low binding' plastics that are marketed as having low protein binding to the surface due to an improved plastic surface. Which technique is used to produce this surface is largely unknown; some suppliers use an improved polymer mix that generates a hydrophilic surface (e.g. Eppendorf), others coat the surface with nano particles or vial walls are treated with strong acids. In the current study, four dif ferent types of 'low binding' microtubes were tested (A, Eppendorf; B, Sarstedt; C, Biozym; D, Corning) and compared to standard PP microtubes (Sarstedt) and standard glass vials (Carl Roth). MC LR and MC LF as representative toxins for high and low recovery, respectively, were chosen for a first screening at a $\mathrm{MC}$ congener concentration of $10 \mu \mathrm{g} / \mathrm{L}$ (Fig. 6A). For MC LR, 'low binding' tubes type A $C$ showed a similarly high recovery compared to glass ves sels, whereas type D provided a slightly lower recovery (Fig. 6A). For MC LF, only 'low binding' tubes type A and B performed well 

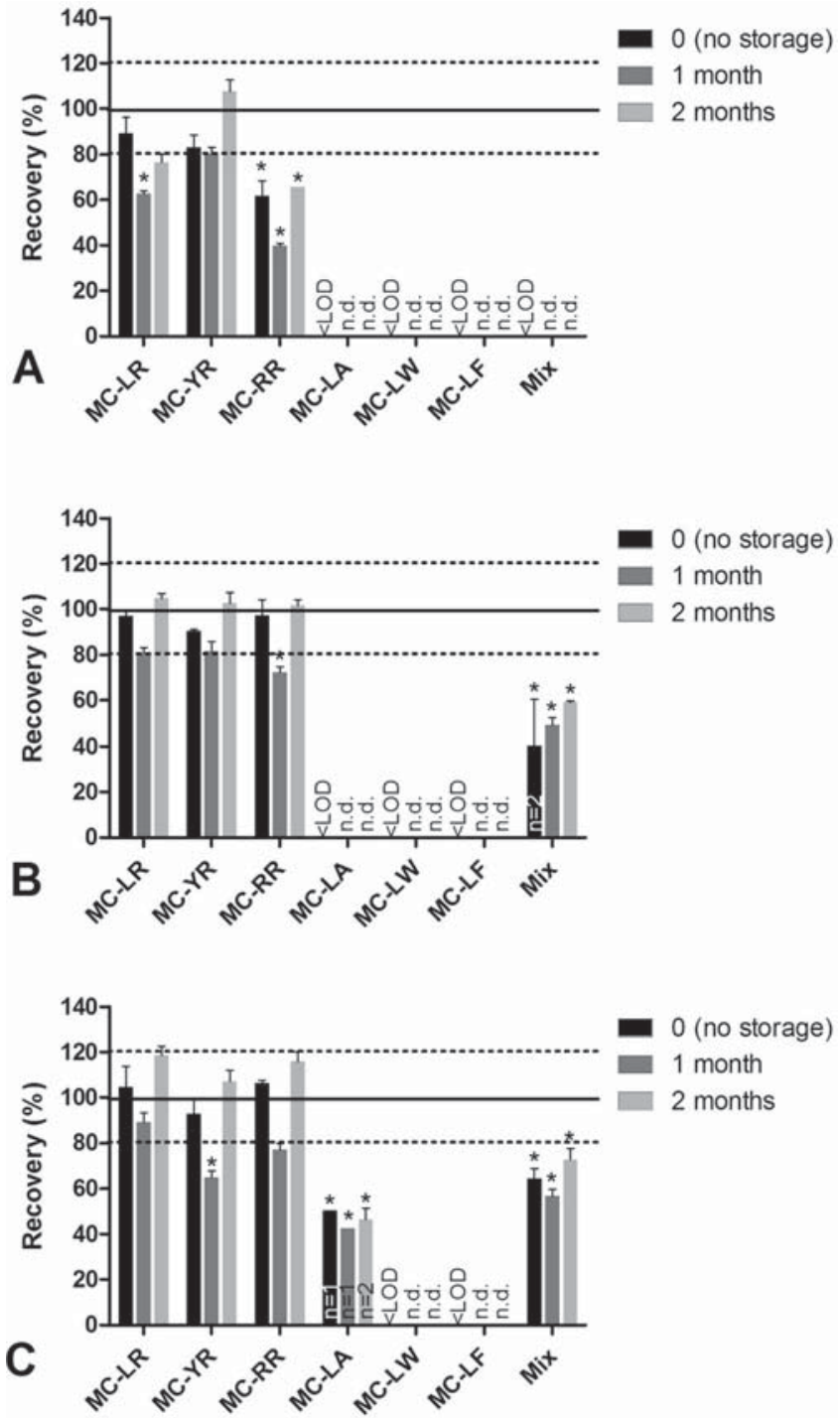

Fig. 5. Recovery of six $M C$ congeners from spiked human serum (standard polypropylene vials) using the Adda-ELISA. Human serum was spiked with $1 \mu \mathrm{g} / \mathrm{L}$ (A), $10 \mu \mathrm{g} / \mathrm{L}$ (B) and $100 \mu \mathrm{g} / \mathrm{L}$ (C) of each MC congener or an equimolar mixture of the six MC congeners to a final total MC concentration of $1 \mu \mathrm{g} / \mathrm{L}(\mathrm{A}), 10 \mu \mathrm{g} / \mathrm{L}$ (B) and $100 \mu \mathrm{g} / \mathrm{L}(\mathrm{C})$. Standard polypropylene vials were used for storage $\left(-20^{\circ} \mathrm{C}\right)$ and processing. Columns represent means from three independent samples $(n=3$; unless stated otherwise) + SEM; dotted lines represent the recovery range (80$120 \%)$. One-way ANOVA with Dunnett's multiple comparison test with $p<0.05(*)$. LOD, limit of detection $=0.15 \mu \mathrm{g} / \mathrm{L}$; n.d., not determined.

and types $C$ and D showed massively decreased recovery (Fig. 6A). Based on these results, 'low binding' tubes type A (Eppendorf) was further tested with all six MC congeners and at three different con centrations (1, 10, $100 \mu \mathrm{g} / \mathrm{L}$ ) (Fig. 6B). Only MC LR, YR and RR provide for acceptable recoveries at the highest $\mathrm{MC}$ concentration employed, whereas the other MC congeners and lower MC concen trations resulted in significantly lower recoveries (Fig. 6B). For MC LA, LW and LF, the loss was even higher at the highest con centrations as these required further dilution to fit into the ELISA detection range, which incipiently increased the number of molec ular contacts to the plastic (Fig. 6B). It must be noted however, that even 'low binding' tubes type A (Eppendorf) provided for a rather inconsistent recovery, especially as MC LF tested in triplicates in the first screening (Fig. 6A) resulted in an acceptable recovery, while the retest using a multiple MC concentration format (Fig. 6B) resulted in the absence of any recovery for the very same
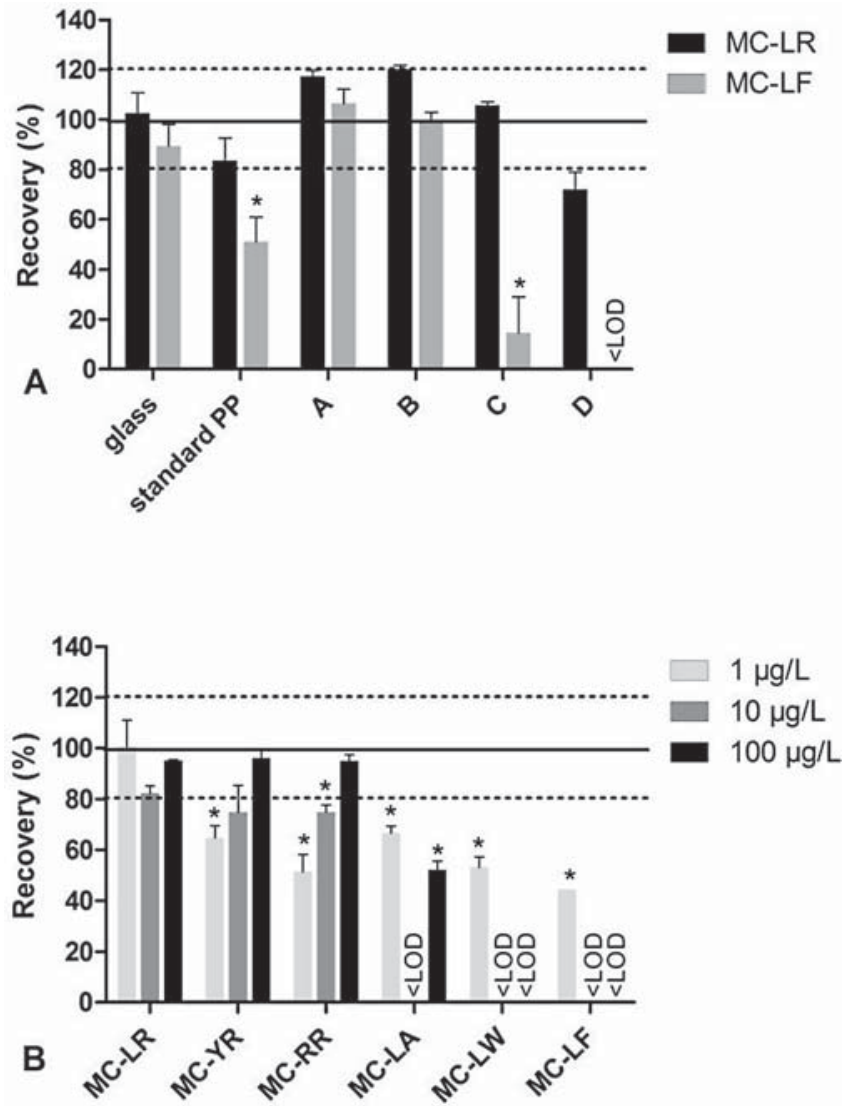

Fig. 6. Low binding polypropylene vial screening (Adda-ELISA). (A) Using human serum spiked with $10 \mu \mathrm{g} / \mathrm{L}$ MC-LR and MC-LF without storage and low binding polypropylene vials from different manufacturers (A, Eppendorf; B, Sarstedt; C, Biozym; D, Corning) compared to glass (Carl Roth) and standard PP vials (Sarstedt). (B) Using human serum spiked with six different MC congeners to a final concentration of 1,10 and $100 \mu \mathrm{g} / \mathrm{L}$ in the low binding vial type A (Eppendorf) without further storage. Columns represent means from three independent samples $(n=3)+$ SEM; dotted lines represent the recovery range $(80-120 \%)$. One-way ANOVA with Dunnett's multiple comparison test with $p<0.05(*)$. LOD, limit of detection $=0.15 \mu \mathrm{g} / \mathrm{L}$.

extraction and analysis methods used. The latter is interpreted as potential high variability of the "coating" of individual low binding PP tubes with the hydrophile polymer mix.

\subsection{Effect of Teflon on the recovery of MCs}

As during the recovery studies, the impression was gained that Teflon caps of the vials could increase loss of MC congeners, this was tested in a separate experimental approach (Fig. 7A and B). Human serum was spiked with $10 \mu \mathrm{g} / \mathrm{L}$ of MC LR, LA or LF, as rep resentative MCs for high, moderate and low recovery, extracted and the extract transferred into standard glass vials with Teflon lined caps. Concurrent controls were established using 5\% metha nol solutions that were spiked with $10 \mu \mathrm{g} / \mathrm{L}$ MC LR, LA or LF and the spiked solutions were directly transferred into the glass vials with the Teflon lined caps. These Teflon lined cap glass vials were then mixed for $2.5 \mathrm{~h}$ either on a horizontal shaker or on an overhead rotator. Irrespective of whether the spiking occurred directly into a $5 \%$ methanol solution or whether the MCs were extracted from human serum, contact with the Teflon lid resulted in a substantial loss of some MCs, especially MC LF, whereas MC LR recovery was not affected (Fig. 7A and B). Interestingly, this effect was more pronounced in extracted samples from human serum when compared to the directly spiked methanol solution. 

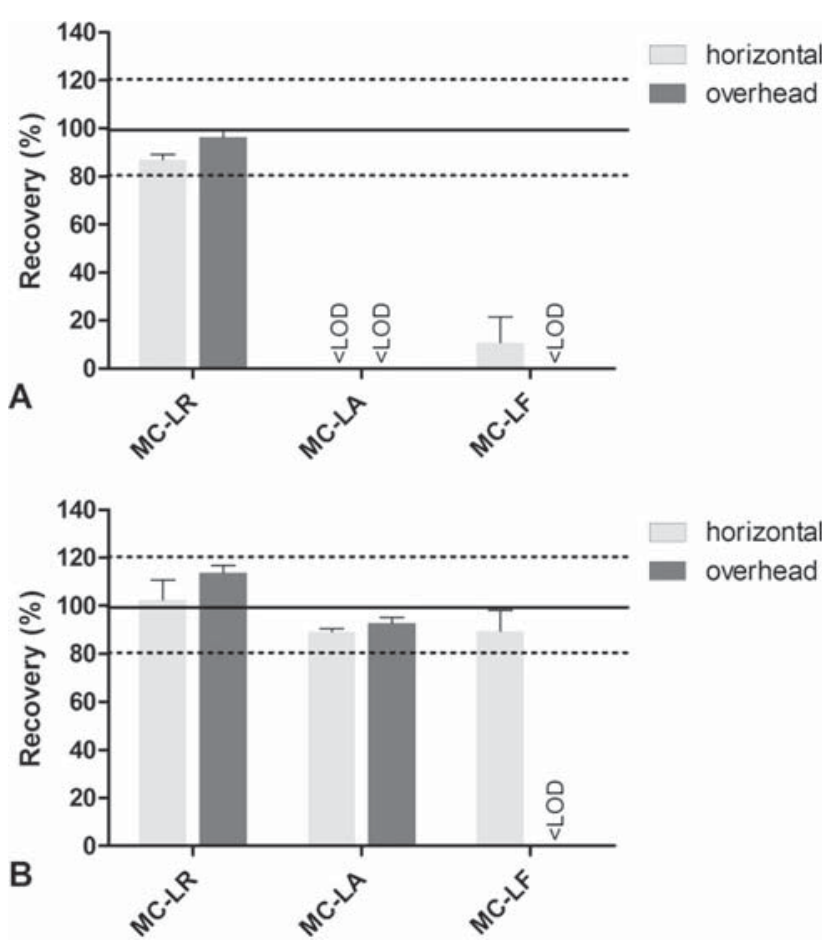

Fig. 7. Effect of Teflon liners on the recovery of MCs (Adda-ELISA). MCs extracted from human serum (A) or MCs in 5\% methanol (B), each spiked with $10 \mu \mathrm{g} \mathrm{MC/L}$ were placed in glass vials with Teflon-lined caps and mixed for $2.5 \mathrm{~h}$ using either a horizontal or an overhead shaker. Columns represent means from three independent samples $(n=3) \pm$ SEM; dotted lines represent the recovery range $(80-120 \%)$. LOD, limit of detection $=0.15 \mu \mathrm{g} / \mathrm{L}$.

Thus the loss in recovery observed for MC LA and LF from the human serum samples may have also been the result of the extrac tion procedure, as suggested earlier.

\subsection{Effect of pipetting on the recovery of individual MC congeners}

In order to complete the study, the effect of repeated pipetting on MC recovery was analyzed using MC LR, LA and LF as repre sentative MC congeners for high, moderate and low recovery, respectively. MCs were spiked into $5 \%$ and $80 \%$ aqueous methanol in standard glass vials followed by an additional 0,5 and 10 pipet ting steps before these samples were analyzed via the Adda ELISA. Pipetting as such had no significant effect on the recoveries of the individual MC congeners (Fig. 8). However, the amount of organic solvent (methanol) used, had a significant effect on the recovery of the more lipophilic MC congeners MC LA and MC LF, whereas this effect was most pronounced for the most lipophilic MC LF (Fig. 8C).

\section{Discussion}

Reliable extraction, handling and detection of microcystins from human tissue, e.g. serum, has been an ongoing issue in con junction with safety and risk assessment of human exposure but also in conjunction with human intoxications with fatal outcome [22]. There are two main parts to this issue, namely (i) what is the loss incurred during the extraction from human tissue (in this case blood serum) for the different MC congeners; and (ii) what is the loss incurred during the handling and storage of MC contami nated material prior to analysis for the different MC congeners. Obviously both questions are strongly related to the chemical properties of the different MC congeners and thus their behavior
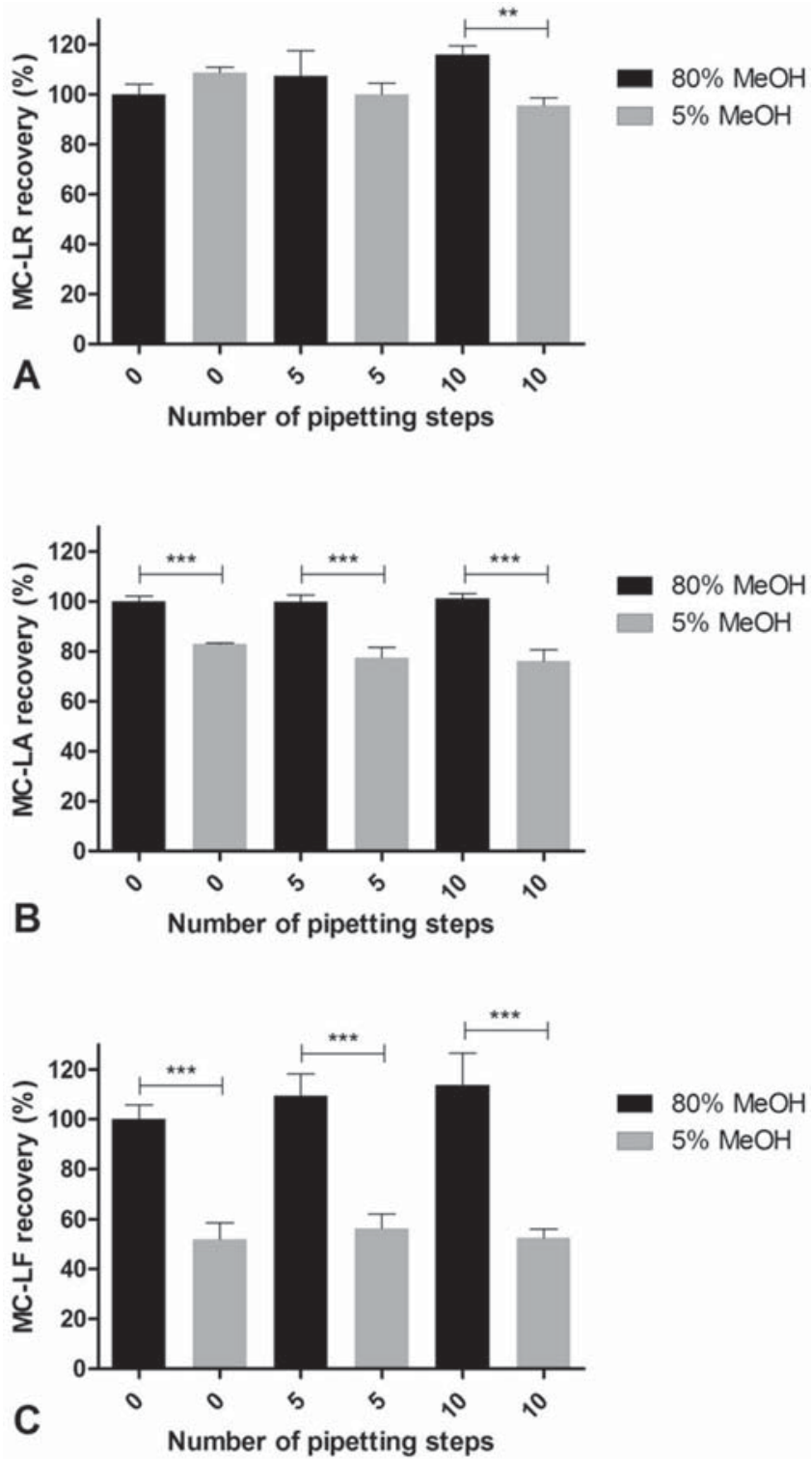

Fig. 8. Effect of pipetting steps and the amount of organic solvent used on the recovery of MCs (analyzed via Adda-ELISA). MC-LR (A), MC-LA (B) and MC-LF (C) were spiked at a concentration of $32 \mu \mathrm{g} / \mathrm{L}$ into $5 \%$ or $80 \%$ aqueous methanol in glass vials and additional pipetting steps were applied to the samples prior to analysis. Samples submitted to ELISA were diluted to $2 \mu \mathrm{g} / \mathrm{L}$ MCs and $5 \%$ methanol before analysis. Values were normalized to the respective $80 \%$ methanol sample without pipetting steps. Columns represent means from three independent samples $(n=3)+$ SD; One-way ANOVA with Tukey's multiple comparison test with $p<0.01$ $(* *)$ and $p<0.001(* * *)$.

in biological and chemical systems. When comparing the struc tures of the six MC congeners employed in this experiment, it becomes quickly apparent that these congeners differ only in their variable $\mathrm{L}$ amino acid moieties (Fig. 1). It thus follows that the chemical behavior (hydrophobicity and surface adhesion capabili ties) of the whole MC molecule would be governed by the bio chemical properties of the two amino acids. The biochemical properties of the amino acids in question: arginine, tyrosine, ala nine, leucine, tryptophan and phenylalanine have distinct differ ences in hydrophobicity, whether these were determined using theoretical calculations, octanol/water partition coefficients, aque ous two phase systems or experimental assays $[23,24]$. Based on the latter analyses, a grading of increasing hydrophobicity of argi nine $\ll$ tyrosine $<$ alanine, leucine $<$ tryptophan $<$ phenylalanine 
can be established. Moreover, the presence of planar aromatic rings of tryptophan and phenylalanine will add an additional factor, i.e. adhesion capabilities [25]. Consequently, the combination of these six amino acids within the two variable $L$ amino acid residues would predict the following grading of increasing hydrophobicity: MC RR < MC YR, MC LR < MC LA < MC LW < MC LF, and increasing adhesion capability: MC RR, MC LA, MC LR $<$ MC YR $\ll$ MC LW $<$ MC LF. The latter theoretical considerations are corroborated by experimentally determined 1 octanol/water partition coeffi cients $(\log P)$ for MC LR, LW and LF of 2.16, 3.46 and 3.56, respec tively $[26,27]$ and more importantly by HPLC [28]. Indeed, HPLC using the chemical interactions (hydrophobic, van der Waals dis persive, dipole dipole, ionic, etc.) of the molecule of interest between the organic mobile and the stationary phase (sorbent) demonstrated an increasing retention time of MCs with increasing hydrophobicity thus resulting in the following elution order: (1) MC RR, (2) MC YR, (3) MC LR, (4) MC LA, (5) MC LW and (6) MC LF, as also confirmed in this laboratory.

In view of the above chemical and structural considerations, it is not surprising that the less hydrophobic MC LR can be recovered from human serum rather robustly even after prolonged storage times (Fig. 3) irrespective of the handling and storage materials (glass or polypropylene) used. Despite this, Hyenstrand and co workers $[14,15]$ reported that MC LR adsorbed to plastic pipette tips leading to a loss of $4.2 \%$ per tip operation. This loss in MC LR recovery was significant when using methanol solutions contain ing $<25 \% \mathrm{MeOH}$ and could not be attributed to other factors such as $\mathrm{pH}$ or salinity of the solution [15] and thus was assumed to stem from surface adsorption of aromatic moieties, e.g. the Adda side chain present in all of the known MC congeners. Thus, if the Adda side chain and adsorption governs the latter reduction in recovery, then other MC congeners with higher hydrophobicity and number of aromatic moieties would result in an even greater loss (lower recovery). Indeed, this was corroborated as shown in Fig. 4, whereby the least hydrophobic MC congeners MC RR, YR, and LR demonstrated acceptable recoveries from human serum irrespective of the handling and storage materials used. In contrast, the more hydrophobic MC congeners demonstrated a nearly acceptable recovery only in glass vials and when analyzed without storage time, whereas use of standard polypropylene vials for han dling and storage (Fig. 5) resulted in absolute absence of any recov ery irrespective of the MC congener concentration originally used. Thus, the hydrophobicity ranking of these six MC congeners corre sponds to the observed degree of MC loss during preparation and analytics in this study. It can be therefore concluded that hydropho bicity and adsorption are indeed the most important factors in low $\mathrm{MC}$ recoveries from labware and that the use of polypropylene lab ware should be avoided whenever possible, including the so called low binding polypropylene vessels (Fig. 6). A previous study dem onstrated promising MC recovery performance of high density polyethylene (HDPE) vials with respect to short term (hours) use [11]. Thus HDPE vials may be an alternative for the centrifugation step, which cannot be performed in the conical glass vials.

Obviously, and as also shown by Hyenstrand and co workers [14,15] for MC LR and in Fig. 8 for the other five MC congeners, increasing the $\mathrm{MeOH}$ concentration will serve to reduce the amount of retention and thus recovery loss for all MC congeners including the most hydrophobic ones. In this context, it is not sur prising that the use of no greater than $5 \% \mathrm{MeOH}$ solutions (Fig. 2) as required for Adda ELISA (Rubio and Kamp, unpublished results) and other commercial ELISAs [13] is not adequate for handling and storing MC congeners. Indeed, a concentration of $80 \%$ is assumed to be optimal for storage, which was already used for the extraction procedure. Consequently, the extraction and storage procedure was modified accordingly and also included the specific omission of Teflon seals as detailed in Fig. 9.
To date only few procedures for the extraction of MCs from human serum or organ samples were described in literature whereby these procedures describe the of $1050 \%(\mathrm{v} / \mathrm{v}) \mathrm{MeOH}$ in water after SPE $[7,10,29]$ for extraction. In most cases, these extracts were further processed and also stored at $-20^{\circ} \mathrm{C}$. No detailed information was given regarding the duration of storage or the materials used for storing the extracts. For example, extracts

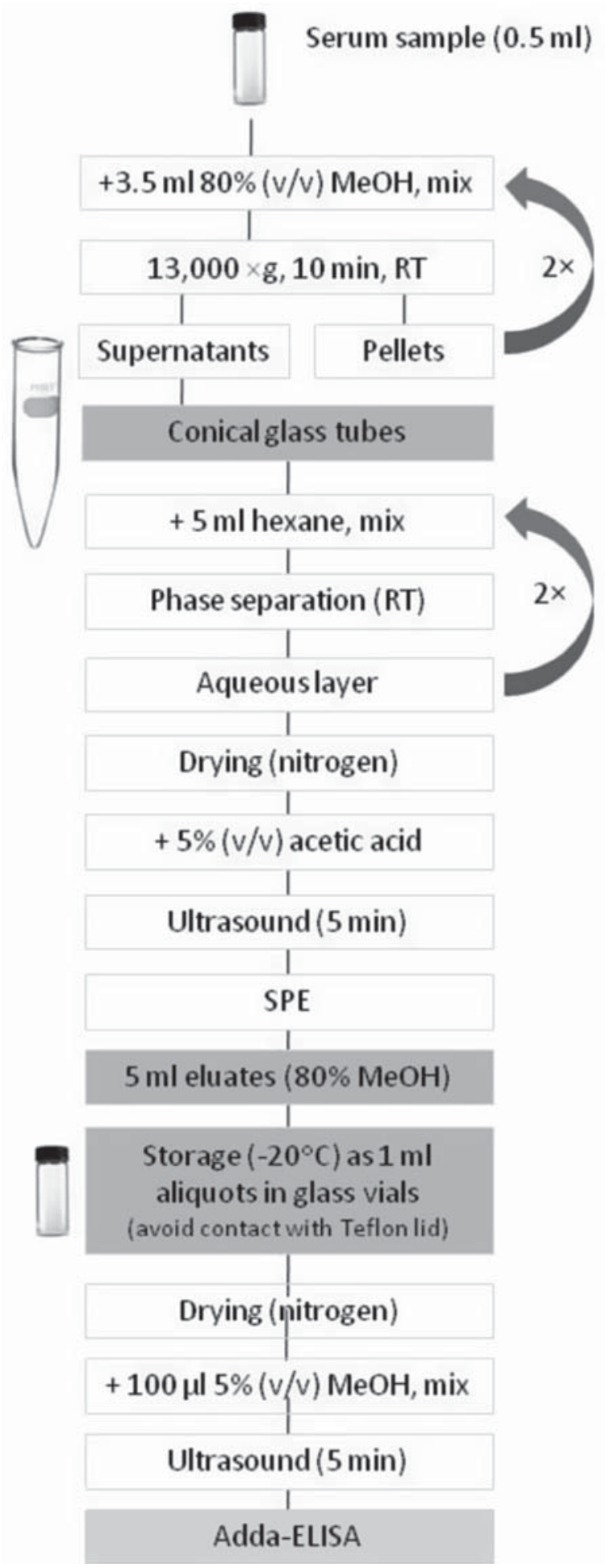

Fig. 9. Proposed future sample preparation procedure prior to ELISA. Restricting the amount of time the sample remains in the $5 \%$ methanol solution prior to ELISA is crucial to the outcome. 
from serum samples collected from Caruaru patients in 1996 pro cessed via $\mathrm{MeOH}$ and SPE extraction and then analyzed via ELISA and by HPLC. MC LR, YR and desmethyl MC LR were reported as the main MC congeners present $[10,29]$. However, these congeners are less hydrophobic compared to other congeners and may be the only congeners among others present in the samples that were recovered by the extraction method employed. Therefore, the pres ence of other congeners cannot be ruled out while the overall amount of MCs may have been severely underestimated due to the limitations of the extraction and storage procedures employed.

Furthermore, the mixture of MC congeners produced by a cyanobacterial bloom can differ dramatically depending on a vari ety of factors, even when the bloom occurs always at the same site [30]. Therefore, humans can be exposed to MC mixtures of different congener compositions. Depending on the extraction method used, particularly the methanol concentration applied, huge deviations from the real exposure level may be observed when primarily hydrophobic MC congeners are present compared to a rather coher ent picture when for example MC LR dominates the mixture. Cor rect estimation of the $\mathrm{MC}$ congeners and their respective concentrations present in a given sample are crucial for proper risk assessment, whether this is before the fact (water, fish tissue or algae supplement samples) or after the fact (post human exposure in serum, full blood, or tissue samples). Consequently, any deviation from correct estimation of the MC congeners and their respective concentrations present will have a major impact on risk assessment.

In summary, it is concluded that ELISA based methods have been proven to be suitable tools for the detection of MCs in water, in cyanobacterial cells and in exposed human, animal and plant materials (more than 200 entries in PubMed since 1989). The pit falls described for MC analysis with the ELISA are primarily associ ated with sample preparation and clean up methodology and thus also apply to other analytical techniques for MC detection beyond the ELISA technology reported here.

\section{Conflict of Interest}

Dr. Dietrich reports grants from Marie Curie International Research Staff Exchange Fellowship within the 7th European Com munity Framework Program (PIRSES GA 2011 295223), during the conduct of the study; In addition, Dr. Dietrich has a patent "Congener independent detection of microcystin and nodularin congeners". Patent \# PCT WO 01/18059 issued to Abraxis LLC. Dr. Rubio is head of Abraxis Ltd and thus manufacturer of the ELISA employed in this study.

\section{Transparency Document}

The Transparency document associated with this article can be found in the online version.

\section{Acknowledgements}

This work was supported by a Marie Curie International Research Staff Exchange Fellowship within the 7th European Com munity Framework Program (PIRSES GA 2011 295223).

\section{References}

[1] S. Merel, D. Walker, R. Chicana, S. Snyder, E. Baurès, O. Thomas, State of knowledge and concerns on cyanobacterial blooms and cyanotoxins, Environ. Int. 59 (2013) 303-327.

[2] T.A. Msagati, B.A. Siame, D.D. Shushu, Evaluation of methods for the isolation, detection and quantification of cyanobacterial hepatotoxins, Aquat. Toxicol. 78 (2006) 382-397.
[3] A.H. Heussner, L. Mazija, J. Fastner, D.R. Dietrich, Toxin content and cytotoxicity of algal dietary supplements, Toxicol. Appl. Pharmacol. 265 (2012) 263-271.

[4] V. Mulvenna, K. Dale, B. Priestly, U. Mueller, A. Humpage, G. Shaw, G. Allinson, I. Falconer, Health risk assessment for cyanobacterial toxins in seafood, Int. J. Environ. Res. Public Health 9 (2012) 807-820.

[5] S. Wood, D. Dietrich, Ouantitative assessment of aerosolized cyanobacterial toxins at two New Zealand lakes, J. Environ. Monit. 13 (2011) 1617-1624.

[6] E.D. Hilborn, W.W. Carmichael, R.M. Soares, M. Yuan, J.C. Servaites, H.A. Barton, S.M.F.O. Azevedo, Serologic evaluation of human microcystin exposure, Environ. Toxicol. 22 (2007) 459-463.

[7] Y. Li, J.A. Chen, Q. Zhao, C. Pu, Z. Qiu, R. Zhang, W. Shu, A cross-sectional investigation of chronic exposure to microcystin in relationship to childhood liver damage in the Three Gorges Reservoir Region, China, Environ. Health Perspect. 119 (2011) 1483-1488.

[8] E.D. Hilborn, R.M. Soares, J.C. Servaites, A.G. Delgado, V.F. Magalhães, W.W. Carmichael, S.M. Azevedo, Sublethal microcystin exposure and biochemical outcomes among hemodialysis patients, PLoS One 8 (2013) e69518.

[9] R.M. Soares, M. Yuab, J.C. Servaites, A. Delgado, V.F. Magalhaes, E.D. Hilborn, W.W. Carmichael, S.M.F.O. Azevedo, Sublethal exposure from microcystins to renal insufficiency patients in Rio de Janeiro, Brazil, Environ. Toxicol. 21 (2006) 95-103.

[10] E.D. Hilborn, W.W. Carmichael, M. Yuan, S.M.F.O. Azevedo, A simple colorimetric method to detect biological evidence of human exposure to microcystins, Toxicon 46 (2005) 218-221.

[11] A.H. Heussner, I. Winter, S. Altaner, L. Kamp, F. Rubio, D.R. Dietrich, Comparison of two ELISA-based methods for the detection of microcystins in blood serum, Chem-Biol. Interact. 223 (2014) 10-17.

[12] J.S. Metcalf, G.A. Codd, Analysis of cyanobacterial toxins by immunological methods, Chem. Res. Toxicol. 16 (2003) 103-112.

[13] J.S. Metcalf, P. Hyenstrand, K.A. Beattie, G.A. Codd, Effects of physicochemical variables and cyanobacterial extracts on the immunoassay of microcystin-LR by two ELISA kits, J. Appl. Microbiol. 89 (2000) 532-538.

[14] P. Hyenstrand, J.S. Metcalf, K.A. Beattie, G.A. Codd, Effects of adsorption to plastics and solvent conditions in the analysis of the cyanobacterial toxin microcystin-LR by high performance liquid chromatography, Water Res. 35 (2001) 3508-3511.

[15] P. Hyenstrand, J.S. Metcalf, K.A. Beattie, G.A. Codd, Losses of the cyanobacterial toxin microcystin-LR from aqueous solution by adsorption during laboratory manipulations, Toxicon 39 (2001) 589-594.

[16] I.R. Falconer, A.R. Humpage, Health risk assessment of cyanobacterial (bluegreen algal) toxins in drinking water, Int. J. Environ. Res. Public Health 2 (2005) $43-50$.

17] K. Harada, K. Matsuura, M. Suzuki, M.F. Watanabe, S. Oishi, A.M. Dahlem, V.R. Beasley, W.W. Carmichael, Isolation and characterization of the minor components associated with microcystins LR and RR in the cyanobacterium (blue-green algae), Toxicon 28 (1990) 55-64.

[18] R.W. Burke, R. Mavrodineanu, Acidic potassium dichromate solutions as ultraviolet absorbance standards, J. Res. NBS 80A (1976) 631-636.

[19] S.J. Hoeger, D. Schmid, J.F. Blom, B. Ernst, D.R. Dietrich, Analytical and functional characterization of microcystins [Asp3]MC-RR and [Asp3, Dhb7]MC-RR: consequences for risk assessment?, Environ Sci. Technol. 41 (2007) 2609-26016.

[20] W.J. Fischer, I. Garthwaite, C.O. Miles, K.M. Ross, J.B. Aggen, A.R. Chamberlin, N.R. Towers, D.R. Dietrich, Congener-independent immunoassay for microcystins and nodularins, Environ. Sci. Technol. 35 (2001) 4849-4856.

[21] EPA, Technology Brief: Immunoassay Test Kits for Microcystins (EPA/600/S/12/ 511), 2012

[22] S.M.F.O. Azevedo, W.W Carmichael, E.M. Jochimsen, K.L Rinehart, S. Lau, G.R Shaw, G.K. Eaglesham, Human intoxication by microcystins during renal dialysis treatment in Caruaru - Brazil, Toxicology 181-182 (2002) 441-446.

[23] N. Gulyaeva, A. Zaslavsky, P. Lechner, A. Chait, B. Zaslavsky, PH dependence of the relative hydrophobicity and lipophilicity of amino acids and peptides measured by aqueous two-phase and octanol-buffer partitioning. J. Pept. Res. 61 (2003) 71-79

[24] H. van de Waterbeemd, H. Karajiannis, N. El Tayar, Lipophilicity of amino acids, Amino Acids 7 (1994) 129-145.

25] J. Björk, F. Hanke, C.-A. Palma, P. Samori, M. Cecchini, M. Persson, Adsorption of aromatic and anti-aromatic systems on graphene through $\pi-\pi$ stacking, J. Phys. Chem. Lett. 1 (2010) 3407-3412.

[26] C.J. Ward, G.A. Codd, Comparative toxicity of four microcystins of different hydrophobicities to the protozoan, Tetrahymena pyriformis, J. Appl. Microbiol. 86 (1999) 874-882.

[27] P. de Maagd, A. Hendriks, W. Seinen, D. Sijm, PH-dependent hydrophobicity of the cyanobacteria toxin microcystin-LR, Water Res. 33 (1999) 677-680.

[28] M. Craig, T. McCready, H. Luu, M. Smillie, P. Dubord, C. Holmes, Identification and characterization of hydrophobic microcystins in Canadian freshwater cyanobacteria, Toxicon 31 (1993) 1541-1549.

[29] W.W. Carmichael, S.M.F.O. Azevedo, J.S. An, R.J.R. Molica, E.M. Jochimsen, S. Lau, K.L. Rinehart, G.R. Shaw, G.K. Eaglesham, Human fatalities from cyanobacteria: chemical and biological evidence for cyanotoxins, Environ. Health Perspect. 109 (2001) 663-668.

[30] D.R. Dietrich, A. Fischer, C. Michel, S.J. Hoeger, Toxin mixture in cyanobacterial blooms - a critical comparison of reality with current procedures employed in human health risk assessment, Adv. Exp. Med. Biol. 619 (2008) 885-912. 